\title{
Lithium-induced asymptomatic dose-related elevation of serum creatine kinase: a
}

\section{case report}

\author{
Mao-Hsiu HuA 1,2
}

https://orcid.org/0000-0002-1752-5984

SHEN-CHIEH CHANG2 ${ }^{2}$

Mong-Liang Lu'2,3

https://orcid.org/0000-0003-3184-6554

1 Department of Psychiatry, Taipei Veterans General Hospital, Taipei, Taiwan.

2 Department of Psychiatry, Wan-Fang Hospital, Taipei Medical University, Taipei, Taiwan.

${ }^{3}$ Department of Psychiatry, School of Medicine, College of Medicine, Taipei Medical University, Taipei, Taiwan.

Received: 09/30/2019 - Accepted: 02/10/2020

DOI: 10.1590/0101-60830000000245

Hua MH et al. / Arch Clin Psychiatry. 2020;47(4):121-2

\section{Dear Editor,}

Lithium has many adverse effects limiting its prescription ${ }^{1}$. Creatine kinase $(\mathrm{CK})$ is released when skeletal muscle cells are damaged. Psychiatric patients with remarkable CK elevations commonly indicate to two life-threatening physical conditions, rhabdomyolysis and neuroleptic malignant syndrome (NMS). Lithium use is related to rhabdomyolysis and NMS2-4, but the association of lithium and asymptomatic CK elevation had not been reported.

$\mathrm{Mr}$. Y is a 17 -year-old man presenting with hyperactivity and agitation when he was brought to emergency department. He had manic symptoms including elevated mood, decreased need of sleep, grandiosity, hypertalkativeness lasting for one week. Upon emergency room visit his blood test showed increased levels of GOT/GPT (208/63 U/L) and CK (8697 U/L). He was admitted to pediatric ward due to rhabdomyolysis and then transferred to psychiatric ward after the condition became stable (CK was $1215 \mathrm{U} / \mathrm{L}$ at the transferring day). He was treated with quetiapine first but manic symptoms were still active despite the dosage reached to $600 \mathrm{mg} /$ day. His serum CK level decreased to $644 \mathrm{U} / \mathrm{L}$ after two-week admission. We had tried to add mood stabilizers for combination treatment, but valporic acid was discontinued due to allergic reaction and carbamazepine was contraindicated because of his positive HLA-B1502 genotype. Then, we prescribed lithium for him. We started with $300 \mathrm{mg} /$ day and gradually titrated to $900 \mathrm{mg} /$ day. He had obvious response to lithium (Young Mania Rating Scale total score from 43 to 12 after the dose reaching $900 \mathrm{mg} /$ day). When monitoring his laboratory parameters and serum lithium concentration, we found the serum CK level increased again after lithium treatment. Further, his serum CK levels were positively correlated to serum lithium levels. Lithium was then discontinued and his serum CK level returned to baseline (the time trends are shown in Figure1). During the period of abnormal CK level, he did not have hyperactivity and other physical complaints, and his associated laboratory data were unremarkable (normal liver, renal function and urine globulin).

Lithium interferences with the collecting tubules and suppress antidiuretic hormone stimulation, which makes its polyuria and polydipsia side effects ${ }^{1}$. Another case report assumed lithium might induce hyperosmolar state 3 . These conditions might induce elevated $\mathrm{CK}$ levels and even rhabdomyolysis. However, our patient did not have the above states. Besides, the positive correlation between lithium and CK concentration was not mentioned before. The concentration-dependent relationship between lithium and CK needs further investigations.
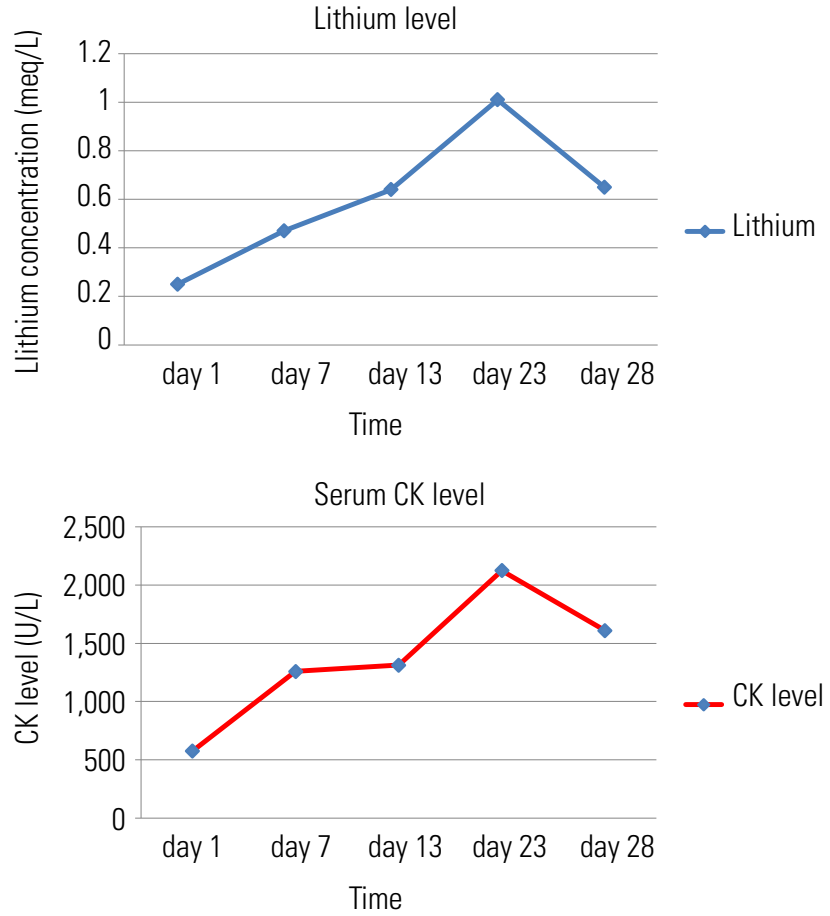

Figure 1. The time trends of lithium and creatine kinase (CK) levels.

Massive Asymptomatic Creatine Kinase Elevation (MACKE) has been discussed in youths using antipsychotics ${ }^{4}$. The article concluded that MACKE is harmless and intensive CK monitor is unnecessary. Whether lithium induced CK elevation is harmless like MACKE or not need more studies to confirm. Furthermore, Gosling et al. found the elevation of serum CK may indicate mania is under controlled5. Our patient also showed obvious response during CK elevation. But using serum CK level to detect lithium treatment efficacy is lack of well-established evidence. Whether lithium induced asymptomatic serum CK elevation is harmful, harmless, or treatment response predictive needs more explorations. 
In addition, studies revealed that higher CK level was found in the manic patients compared with the depressed ones and the lack of correlations between CK level and motor activity ${ }^{6}$. Further studies need to explore the state-dependent differences in CK levels.

\section{Financial support}

None.

\section{References}

1. Gitlin M. Lithium side effects and toxicity: prevalence and management strategies. Int J Bipolar Disord. 2016;4:27.

2. Voros V, Osvath P, Fekete S, Tenyi T. Elevated serum creatine kinase levels in psychiatric practice: differential diagnosis and clinical significance:
A brief, practical guideline for clinicians. Int J Psychiatry Clin Pract. 2008;12(2):147-50.

3. Bateman AM, Larner AJ, McCartney SA, Rifkin IR. Rhabdomyolysis associated with lithium-induced hyperosmolal state. Nephrol Dial Transplant. 1991;6(3):203-5.

4. Masi G, Milone A, Viglione V, Mancini A, Pisano S. Massive asymptomatic creatine kinase elevation in youth during antipsychotic drug treatment: case reports and critical review of the literature. J Child Adolesc Psychopharmacol. 2014;24(10):536-42.

5. Gosling R, Kerry RJ, Owen G. Creatine phosphokinase activity during lithium treatment. Br Med J. 1972;3(5822):327-9.

6. Segal M, Avital A, Drobot M, Lukanin A, Derevenski A, Sandbank S, et al CK levels in unmedicated bipolar patients. Eur Neuropsychopharmacol. 2007;17(12):763-7. 